\title{
ARZNEIMITTELALLERGIKER
}

\section{Jeder Dritte schluckt irgendwann wieder die falschen Pillen}

Jeder dritte Arzneimittelallergiker nimmt einige Jahre nach der Diagnose wieder ein Medikament ein, dass das auslösende Allergen enthält, haben Forscher der Hautklinik der Universitätsmedizin Göttingen festgestellt. Ein Teil der Allergiker vergisst, auf welchen Stoff er allergisch reagiert. Andere machen sich nicht die Mühe, den Beipack- zettel von Medikamenten zu prüfen. Was die Göttinger Forscher besonders irritiert: Es gibt auch Ärzte, die sich über die Empfehlungen der Allergologen hinwegsetzen und den Allergikern das unverträgliche Medikament erneut verordnen.

http://www.lifepr.de/pressemeldungen/aerzteverband-deutscher-allergologen-ev-aeda/boxid/216679

\section{HITZEFOLGEN BEIM HOCHLEISTUNGSSPORT}

\section{Pfaffenmilch macht den Darm dicht}

\begin{abstract}
Langstreckenläufer, die bei großer Hitze unterwegs sind, kennen das Problem: Irgendwann streikt der Darm. Bei körperlicher Anstrengung in heißer Umgebung steigt die Permeabilität der Darmwand um bis zu $250 \%$ an. Um zu verhindern, dass der Organismus mit Toxinen aus dem Darm überschwemmt wird, stellt sich Durchfall ein. Sportliche Höchstleitungen lassen sich unter diesen Umständen nicht erzielen.

Forscher der London School of Medicine and Dentistry haben ein einfaches Mittel entdeckt, mit dem sich die Darmpermeabilität wieder reduzieren lässt: Kolostrum von Kühen. Das ist die Milch, die Kühe in den ersten Tagen nach dem Kalben

abgeben (auch bekannt als Vormilch, Biestmilch, Pfaffenmilch oder Kuhpriester).

Kolostrum bildet im Darm von neugeborenen Kälbern einen Schutzfilm. Diesen Effekt kann man auch beim Menschen nutzen. Bei Sportlern, die 14 Tage lang täglich Kolostrum getrunken hatten, nahm die Erhöhung der Darmpermeabilität unter Anstrengung um $80 \%$ ab. Kolostrum könnte möglicherweise auch Folgen eines Hitzschlags mindern, meinen die Londoner Forscher. Bei uns ist Kolostrum nur als Diätmittel zugelassen.

American Journal of Physiology - Gastrointestinal and Liver Physiology, März 2011
\end{abstract}

\section{VENÖSE THROMBOEMBOLIE}

\section{Wie hoch ist das Rezidivrisiko?}

Nach einer Venenthrombose oder einer Lungenembolie ohne Provokation sind Männer besonders gefährdet, ein Rezidiv zu erleiden. Zu diesem Ergebnis kommt eine Metaanalyse mit 2554 Patienten mit einer ersten venösen Thromboembolie (VTE). Die Inzidenz eines erneuten Ereignisses lag nach einem Jahren bei 5,3\% (Frauen) und

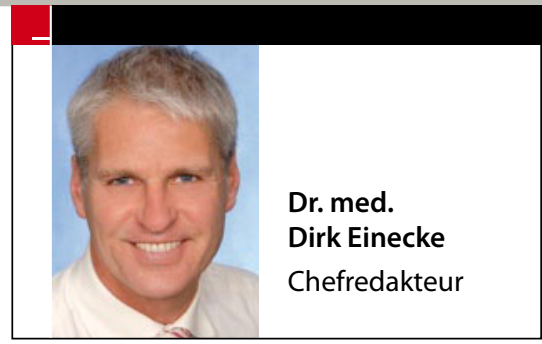

SCHLAGANFALL

\section{Die Gefahr steigt mit den Triglyzeriden}

Triglyzeride sind für das Schlaganfallrisiko möglicherweise noch bedeutsamer als Cholesterin. In der Copenhagen City Heart Study, in der 7579 Frauen und 6372 Männer bis zu 33 Jahre lang beobachtet wurden, zeigte sich, dass Frauen mit (nicht im Nüchternzustand gemessenen) Triglyzeridspiegeln über $5 \mathrm{mmol} / \mathrm{l}$ ein fast vierfach höheres Risiko für einen Schlaganfall haben als Frauen mit Spiegeln unter $1 \mathrm{mmol} / \mathrm{l}$. Bei den Männern erhöhte sich das Risiko um das 2,3-Fache. Das LDL-Cholesterin ging in dieser Studie erst ab stark erhöhten Werten (über $9 \mathrm{mmol} / \mathrm{l}$ ) bei Männern, nicht jedoch bei Frauen mit einem erhöhten Schlaganfallrisiko einher.

Ann Neurol 2011; DOI: 10.10002/ana.22384

www.SpringerMedizin.de

Top 3 aus der Inneren Medizin:

Diese Artikel waren auf SpringerMedizin.de in den letzten Tagen die Lese-Hits im Bereich Innere Medizin:

_ Emphysem: Ventile sollen frischen Wind in die Lunge bringen (335632)

_ Diabetes im Anmarsch? Schon $\mathrm{ab} \mathrm{HbA} \mathrm{H}_{1 \mathrm{c}}$ von fünf Prozent droht Zuckeralarm! (334044)

- Vitalparameter im Blick: Neues Intensiv-Monitoring für die Endoskopie (337200)

Die Artikel finden Sie, indem Sie die ID-Nummer (in Klammern) in die Suche eingeben. 